\title{
Attentional strategies for studying scientific texts
}

\author{
DIANA DEE-LUCAS and JILL H. LARKIN \\ Carnegie-Mellon University, Pittsburgh, Pennsylvania
}

\begin{abstract}
Content-area novices develop rules as to what types of information (e.g., definitions, facts, equations) are important in texts (Dee-Lucas \& Larkin, 1988). The study reported here indicates that these rules influence text learning. Experts and novices read and recalled science texts. Reading times and recall for different types of content were compared for the two groups. Results indicate that novices' importance rules function as part of novices' control schema during reading, influencing their attentional processes and the resulting representation formed for the text. This was evident in qualitative differences between experts and novices in their attentional patterns and text recall. The study also found that the number of passage readings and the passage topic have a greater influence on the reading times of experts, but both groups adjust processing time according to the hierarchical level of the passage content. The findings are discussed in terms of their implications for novices learning from texts.
\end{abstract}

Recent research on text comprehension has explored how readers' knowledge structures interact with text content in determining what is learned. These knowledge structures range from very general structural schemata for a writing genre (e.g., story grammars) to domainspecific representations of a content area (e.g., the goal structure for baseball). They all aid readers' comprehension by guiding attention to important and relevant content, facilitating encoding of that information into existing knowledge structures, and providing retrieval cues. Thus a reader's prior knowledge plays an important role in determining what is learned from text.

The present study examined how the primitive knowledge structures of novices influence attention and learning from unfamiliar scientific texts. Most research on knowledge effects has considered well-developed stable knowledge structures, such as story schema, or expert knowledge. Relatively little is known about the more tentative knowledge structures developed by novices as they learn about a content domain. These knowledge structures are difficult to characterize due to their transient nature (i.e., they are continually updated and modified as more knowledge is acquired) and the potential variability among people beginning to learn about a content area. However, Dee-Lucas and Larkin $(1986,1988)$ have begun to describe the knowledge structures of novices learning physics. They have found that people learning physics form rules that indicate what types of easily recognizable information (e.g., definitions, facts, equations) are important in physics texts. These rules are part of novices' developing knowledge structure for the domain of physics.

A version of this paper was presented at the 1985 American Educational Research Association meeting. This research was supported by Army Research Institute Contract MDA 903 85K 0180 awarded to the authors. We would like to thank Susan Stauffacher and Stephanie Smith for their help in data collection. Address reprint requests to Diana DeeLucas, Department of Psychology, Carnegie-Mellon University, Pittsburgh, PA 15213.
The current study investigated the extent to which these importance rules influence novices' attention and learning when they are studying physics texts (i.e., repeatedly rereading passages to prepare for a test on content). The research examined whether novice rules are used to guide attention during reading, how the use of these rules interacts with overall passage familiarity in modifying attention during repeated study trials, and whether the resulting attentional differences are reflected in what is remembered afterward. To the extent that novice importance rules control text processing, the nature of these rules (i.e., their initial accuracy and how they change with additional knowledge) has important implications for the efficiency with which novices learn from texts, and how this efficiency can be increased.

Although recent research has emphasized the importance of domain-related schematic knowledge in text comprehension, readers seem to be able to identify important content in texts about unfamiliar topics without using this type of knowledge. Kieras (1980, 1985; Kieras \& Bovair, 1981) showed that readers can identify thematic information in texts by using surface-level text features (e.g., the topic-comment structure of sentences, passage organization, signaling phrases) and a limited, or "shallow," understanding of the semantic relations among the content (e.g., using semantically entailed information from familiar terms to infer the nature of unfamiliar terms). Thus readers seem to be able to identify the main points in unfamiliar technical texts without relying on knowledge about the content domain by using superficial characteristics of the text structure and semantics. Kieras (1985) argued that domain-specific schemata, therefore, are not particularly important for identifying the important content in technical prose.

However, Dee-Lucas and Larkin $(1986,1988)$ showed that domain-specific rules are used by novices in assessing importance in texts. They found that content-area novices (i.e., beginning-level students) develop rules 
about the relative importance of different categories of information in a domain. These rules are domain specific in that naive subjects (i.e., subjects without domain training) do not possess these rules. Dee-Lucas and Larkin (1988) demonstrated this rule use in a series of experiments in which they manipulated the category membership of information without altering its substantive content and had subjects varying in expertise judge its importance. They found that naive subjects judged the same content as equal in importance regardless of its sentence-category membership. Similarly, experts also judged importance independently of category membership. However, novices altered their assessment of the importance of the passage content according to its category. For example, they judged the same content as more important when it was presented as a definition than as a fact. Thus, although naive, expert, and novice subjects are all able to identify definitions and facts, category membership is only relevant to the novices in assessing importance; that is, only novices think that one category type is more important than another. This is because novices have developed rules regarding the importance of the different categories of information as a result of their limited instruction in the content domain.

These novice importance rules can be thought of as the rudimentary beginnings of a content schema. A content schema indicates how information in a content area is typically organized, including what is important in that domain. These rules are part of novices' evolving content schema, in that they define in part what novices view as important. Sentence category is irrelevant for people without domain training because they lack a content schema and do not have strong expectations about what types of information are important. Sentence category is also irrelevant for experts, who have rich content schemas that allow them to judge importance directly. An evolving novice schema would consist of only a few category rules that specify the importance of different information types. However, with additional knowledge regarding the relative importance of information within type categories, these rules could be refined and develop into a differentiated expert knowledge system. Because novice knowledge structures are primitive, with only a few general rules, novices systematically misidentify the important content in domain-related texts. Comparisons of experts' and novices' judgments of what is important to learn in natural texts indicate that novices do not discriminate among the important and less important content within type categories to the same degree as do experts (Dee-Lucas \& Larkin, 1986).

The current study was an attempt to determine whether the primitive content schema of novices (i.e., sentencetype rules for judging importance) is used by novices in determining what is important as they read, as indicated by the amount of processing time given to different types of information. Reading times for different types of content were examined to see if novice rules are reflected in differential attention given to different category types.
Additionally, the amount of information recalled from different category types was examined to see if level of recall varies with the judged importance of the category, that is, if attentional differences produced by importance rules also are reflected in qualitative differences in recall.

Kieras's (1980, 1985; Kieras \& Bovair, 1981) findings, along with the results of research on novice importance rules, indicate that novices have available several sources of information for judging importance: domainindependent properties of the text structure and content, and primitive domain-specific schemata arising from limited experience with the text domain. Given that novices have available alternative sources of information for judging importance, they might vary the degree to which they rely on these sources when studying a text (i.e., repeatedly rereading a passage to learn its content). Novices might be more likely to rely primarily on objective text-based cues on the initial reading of an unfamiliar passage, and to identify the main ideas using rhetorical indicators of importance. Once they were familiar with structural characteristics of the passage (i.e., its overall organization and emphasis), they could then begin to make more use of their schematic knowledge (i.e., importance rules) on subsequent passage readings and concentrate on parts of the text defined as important within this schema.

In the current study, we investigated this possibility by having subjects read each passage three times, examining reading times for each reading trial. The experimental situation was designed to be similar to a study situation. The subjects were told that they were to read each passage three times and that they would receive a test on its contents. Reading times for initial and subsequent readings were compared to determine whether importance rules interact with overall passage familiarity in controlling attention.

This study focused on attentional and recall differences for definitions and facts, two easily recognized types of information that are common and important in scientific texts. The results of earlier research using naturalistic texts indicate that both novices and experts judge definitions as more important to learn than facts (Dee-Lucas \& Larkin, 1986). However, novices are even more likely than experts to judge definitions as important and facts as unimportant. ${ }^{1}$ Thus novices are missing some important facts and judging as important some less important definitions. This indicates that novices do not distinguish between the important and unimportant content within these type categories. As noted earlier, novices (but not experts) judge definitions as more important than facts, even when the content of the two sentence types is held constant in experimental texts (Dee-Lucas \& Larkin, 1988).

Because the definitions and facts in the passages used in the current study differed in content, they varied in syntactic complexity and familiarity. Content familiarity has been shown to influence both reading time (Graesser, Hoffman, \& Clark, 1980; Johnson \& Kieras, 1983) and recall (Chiesi, Spilich, \& Voss, 1979; Johnson \& Kieras, 1983; Spilich, Vesonder, Chiesi, \& Voss, 1979). 
Familiarity and syntactic complexity were controlled in the data analyses with individual subject ratings. These were performed after the subjects had finished reading each passage and completed a free recall test on its content. Each subject then rated the target sentences according to (1) how familiar he/she had been with the sentence content prior to reading the passages, and (2) how comprehensible the sentences were independent of the familiarity of the content. These ratings were used in the analysis of the reading times to control for differences due to these two variables. ${ }^{2}$ The familiarity ratings were also used as a covariate in the analyses of the recall data.

The influence of passage structure on reading times for definitions and facts was also controlled in this study. DeeLucas and Larkin (1986) found that the difference in the judged importance of the two sentence types varied with the hierarchical level of the content. To determine whether importance rules and text structure interact in influencing attention, we analyzed the organizational structure of the passages in this study into a heirarchy, and classified definitions and facts according to their hierarchical level. The reading times were analyzed as a function of both information type and hierarchical level to determine if the difference between the time spent on definitions and facts varied with their structural importance. This analysis also provided an opportunity to examine general levels effects in reading time.

\section{METHOD}

\section{Stimulus Materials}

The two passages were those used by Dee-Lucas and Larkin (1986) in their research comparing expert and novice importance ratings. The topics were work and energy, and fluid statics. They were 44 and 47 sentences in length. Each passage had been constructed so that it contained 18 target sentences, 9 definitions and 9 facts. The definitions described a concept or construct in terms of a given set of characteristics. The facts presented properties of constructs or substances, such as "The amount of force produced by a fluid varies with surface area," "Atmospheric pressure also varies from day to day due to changes in the air temperature," and "The pressure exerted by the fluid on each part of the surface of this submerged body does not depend on the material that the body is made of."

The definitions and facts were classified according to their level in the passage structure. The structural analysis was performed at the sentence level rather than the more commonly used proposition level (Kintsch, 1974; Kintsch \& van Dijk, 1978; Meyer, 1975). This is because the dependent measures used in this and prior research on novice importance rules have involved complete sentences. The structural analysis (also reported in Dee-Lucas \& Larkin, 1986) produced a hierarchy, with the major points of the passages occurring at the top levels and the more detailed information modifying these points occurring at the lower levels. This analysis was performed by first selecting from the passages the main topics or concepts and placing them at the top of the hierarchy. All sentences whose primary content modified these top-level statements were placed at the next level; information modifying second-level content was placed at the third level. Second- and third-level sentences consisted of examples, derivations (i.e., information implied by higher level content), explanations, subtopics, preconditions (i.e., conditions necessary for a rule, principle, or fact to hold true), attributes, and properties. The target sentences were six facts and six definitions occurring at each of the first three hierarchical levels of the passage hierarchies. Importance rating data reponted in DeeLucas and Larkin (1986) indicate that the judged importance of the target sentences is related to their hierarchical levels, as specified by this analysis procedure. One of the passages is included as an Appendix, with the target definitions and facts noted, along with their hierarchical levels.

\section{Subjects}

The expert group consisted of 18 subjects who had completed at least 1 year of graduate study in physics. The novice group was composed of 18 undergraduates who had completed two semesters of coursework in physics at the college level. Novices with this level of physics training were selected to ensure that the novice group had had enough exposure to physics to have developed informationtype rules, but had not approached the expert level in training. These subjects matched in level of expertise the experts and novices used in earlier research on novice importance rules (Dee-Lucas \& Larkin, 1986, 1988).

\section{Procedure}

The subjects were told that the purpose of the experiment was to find out what types of information people find easy and difficult to understand in physics texts. They were told that they would read each passage three times and then complete a test on its contents. For the first reading, they were to read the passage to find out what it was about and its main points. The second and third times, they were to read more carefully in preparation for the exam. These instructions were given to reduce the potential variability in how subjects interpreted the study task.

The subjects were also told that the type of test that they would receive might vary for different passages. This was done so that readers would not have any strong expectations about what types of information they would be tested on, and so that the free recall test after the first target passage would not influence their reading strategy for the second target passage. To help maintain this expectation of varied test types, different types of tests were given after each of the practice passages. The goal was to force readers to rely on their own content schema for the domain of physics in deciding what was important to learn in the passages.

The passages were presented on a video display terminal one sentence at a time, with subjects controlling the sentence presentation rate by pressing a button. The study was conducted in two sessions. In each session, the subjects read one practice passage and one target passage. The order of presentation of the target passages was counterbalanced. After the first practice passage had been read three times, the subjects were asked to rate their overall familiarity with the passage content on a scale from 1 to 5 , and then complete a multiple-choice test. After reading the target passage three times, the subjects again rated their overall familiarity with the passage topic and then were asked to recall the passage. When they had finished the free recall, they rated the individual target sentences on a 5-point scale according to how familiar they had been with the sentence content prior to reading the passage, and how easy it was to understand the sentence independent of its familiarity. The procedure for the second session was the same. However, the subjects were told that they would be given different types of tests in this session. After completing the readings of the practice passage, they completed a fill-in-the-blank test. After reading the target passage three times, they again completed a free recall test, and then rated the target sentences for their familiarity and comprehensibility. 


\section{RESULTS}

\section{Familiarity Ratings}

An analysis of variance was performed on the familiarity ratings indicating the subjects' overall knowledge of the passage topics. The results indicate significant main effects for group $[F(1,35)=48.37, M S e=24.16$, $p<.001]$ and passage $[F(1,35)=12.76, M S e=3.42$, $p<.001]$. The experts were more familiar with both passages $(M=1.28)$ than were the novices $(M=2.42)$. Both groups were more familiar with work and energy $(M=1.65)$ than fluid statics $(M=2.08)$.

\section{Reading Times}

The individual sentence reading times for definitions and facts were analyzed with a multiple linear regression performed on the $\log$ of the times expressed in milliseconds. A regression analysis was performed on individual sentence reading times so that each subject's familiarity and comprehensibility ratings for each sentence could be entered individually, and so differences in sentence length (i.e., number of propositions) could also be included. One data point was identified as an outlier through a normal probability plot of the residuals for the regression model, and this reading time was eliminated from the analysis. The resulting data set contained a total of 3,887 reading times.

The factors included in the analysis were passage (fluid statics, or work and energy), sentence type (definition or fact), level in the passage hierarchy (first, second, or third), comprehensibility and familiarity ratings, number of propositions, and reading trial (first, second, or third reading). Additionally, several interactions were coded in the analysis as the product of the corresponding main effect variables. The type $\times$ group and type $\times$ trial interactions were included in accordance with the study's hypotheses to see if interactions between these variables contributed to variance in the reading times. Also included was the group $\times$ trial interaction to account for variance due to differences between groups in the degree to which they varied their overall reading speed across trials. The passage $\times$ group interaction was entered because preliminary analyses indicated that the main effect of passage was greater for one group than the other. Finally, the level $X$ passage interaction was included because the magnitude of the effect of level varied with passage. Because the analysis was performed on the individual reading times for each subject on each target sentence, subjects were also included as a categorical variable. The regression coefficients for this model, excluding the subject variables, are presented in Table 1.

Sentence type effects. The positive coefficient for sentence type indicates that, averaged across reading trials, readers spent more time on definitions than on facts. However, the type $\times$ group interaction coefficient indicates that the size of this difference varied with level of expertise. Figure la shows the contributions of group and type to the estimated reading times for the first reading of the passages. These are the predicted reading times for the first reading trial for sentences consisting of eight propositions, which is the average length of the target sentences in this study. ${ }^{3}$

As can be seen in Figure 1a, the difference in the estimated times for definitions and facts is greater for novices than experts, with novices spending more time on definitions and less time on facts compared with experts. This is consistent with the type $\times$ group interaction found by Dee-Lucas and Larkin (1986) with importance judgments and summaries. It suggests that novice importance rules are reflected in attentional processes, in that novices differentiate between definitions and facts more than experts do in both judged importance and reading time.

The type $\times$ trial parameter indicates that the difference in the time spent on definitions and facts also varied with reading trial. The main effect of trial shows that the time spent on both definitions and facts decreased with each

Table 1

Parameter Estimates and Standard Errors for a Regression Model

of the Log of the Reading Times.(in msec) on Definitions and Facts*

\begin{tabular}{lccc}
\hline \multicolumn{1}{c}{ Variable } & Coefficient & $\begin{array}{c}\text { Standard } \\
\text { Error }\end{array}$ & $\begin{array}{c}\text { Standard } \\
\text { Regression Coefficient }\end{array}$ \\
\hline Intercept & 3.510 & - & - \\
Familiarity & .030 & .004 & .106 \\
Comprehensibility & .027 & .004 & .095 \\
Propositions & .037 & .001 & .418 \\
Group & -.063 & .021 & -.104 \\
Type & .072 & .019 & .120 \\
Type $\times$ Group & .032 & .014 & .045 \\
Trial & -.068 & .007 & -.184 \\
Type $\times$ Trial & $-.017 \dagger$ & .008 & -.065 \\
Group $\times$ Trial & .020 & .008 & .076 \\
Level & -.026 & .007 & -.071 \\
Level $\times$ Passage & .022 & .009 & .082 \\
Passage & -.162 & .019 & -.268 \\
Passage $\times$ Group & .060 & .014 & .086 \\
\hline$R^{2}=.52 ;$ multiple $R=.72$. & $\dagger p<.04$; all other coefficients significant at $p<.02$ \\
level. & & &
\end{tabular}


(a)

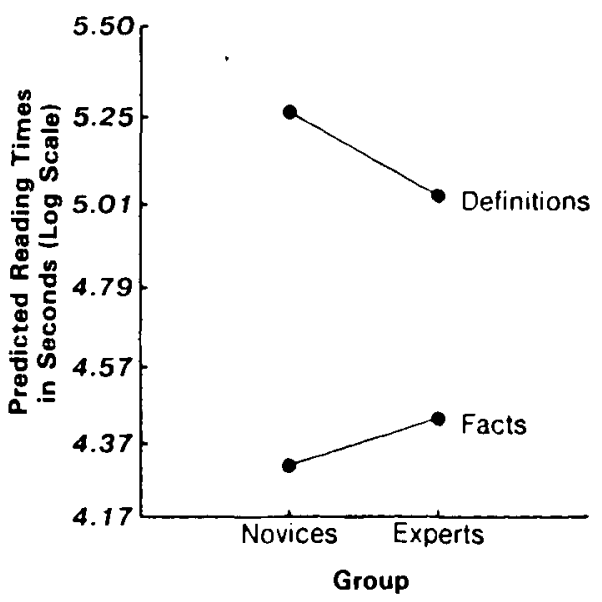

(b)

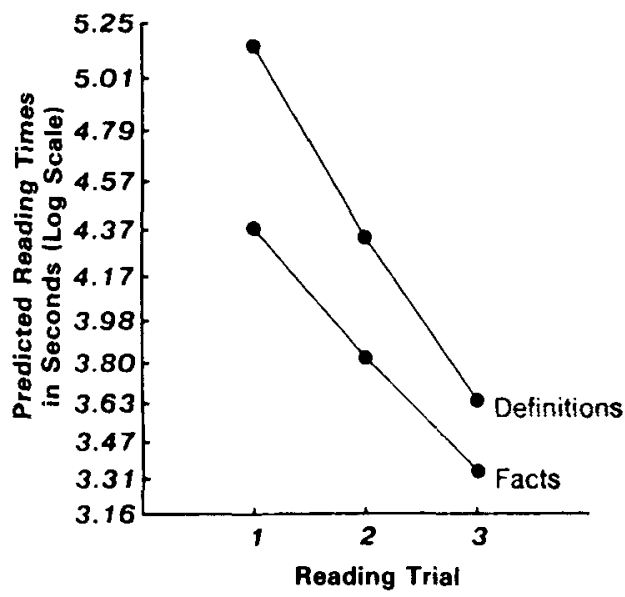

(c)

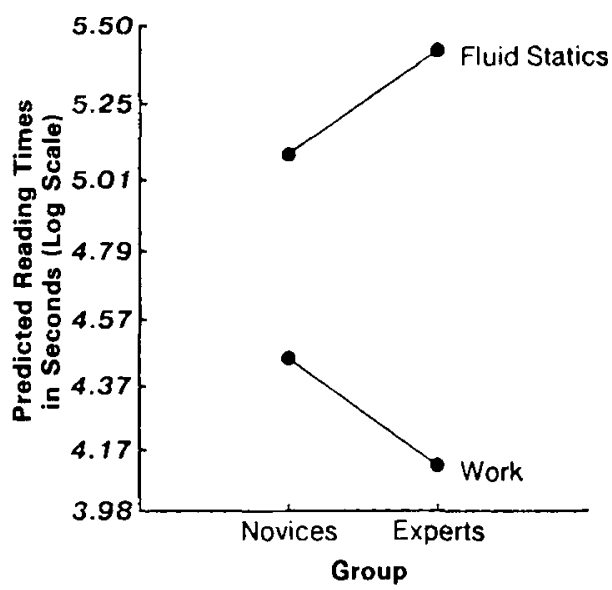

Figure 1. Estimated reading times for the initial reading of the passages, as determined by analyses of (a) group and sentence type, (b) reading trial and sentence type, and (c) passage and group. rereading of the passage. However, the type $\times$ trial estimate shows that the decrease in reading time was greater for definitions than facts. This is shown in Figure lb. This suggests that the greater processing time given to definitions than to facts decreased as the definitions were more thoroughly learned with each subsequent reading trial.

Group differences. Although experts read the target sentences more quickly than did novices, differences in reading speed between the two groups interacted with trial. The parameter for the group $x$ trial interaction indicates that the decrease with each rereading (the main effect of trial) was more rapid for experts than for novices. Thus the difference between the reading times of the experts and the novices increased with each rereading because the experts decreased their reading times over trials more rapidly than did the novices. This may reflect greater confidence on the part of the experts that the subject matter had been sufficiently learned for the anticipated test.

Expert and novice reading times also varied with passage, as indicated by the positive parameter for the group $X$ passage interaction. Readers in both groups spent more time on target sentences in the fluid statics passage (the less familiar passage) than in the work and energy passage. However, the group $\times$ passage estimate indicates that the difference between the amount of time spent on the target sentences in the two passages was greater for the experts than the novices. This interaction is shown in Figure 1c, which indicates that experts spent more time on fluid statics and less time on work and energy than did the novices. Thus the experts discriminated between the two passages to a greater degree than did the novices in terms of adjusting reading times.

Level effects. The parameter estimate for level indicates that readers spent more time on definitions and facts at the higher levels of the passage hierarchy, and less time on lower level sentences. However, the negative parameter for the level $\times$ passage interaction indicates that this effect was greater for the fluid statics passage than for the work and energy passage.

Other variables. Comprehensibility, familiarity, and number of propositions all had the expected effect on sentence reading times. The positive parameters for these variables indicated that readers spent more time on sentences that were less comprehensible, that were less familiar, and that contained more propositions. The fact that the comprehensibility and familiarity variables were significantly related to the variance in reading times suggests that subjects were able to adequately assess preexperimental knowledge and syntactic complexity after reading the passages several times. The individual sentence comprehensibility and familiarity ratings correlated .18 .

\section{Free Recalls}

The free recalls were scored at both the proposition level and the sentence level for the number of target sentences 
and target sentence propositions recalled correctly and incorrectly. A correctly recalled sentence was defined as one in which all the core propositions were recalled accurately. Core propositions were considered to be the propositions expressing the main idea of the sentence. Partial credit was given for recall of sentences having more than one main idea. To receive partial credit, the subject had to recall all of the core propositions for at least one idea. A correctly recalled proposition was either one that was recalled verbatim or one that included substitutions that represented the gist of the proposition elements. Twelve of the 36 recalls were scored by a second scorer, and interscorer reliability was assessed. There was $95 \%$ agreement between the two scorers on the proposition scoring for the recalled sentences.

The free recalls were analyzed with an analysis of covariance (ANCOVA) performed on the mean number of target sentences recalled and on the proportion of total target sentence propositions recalled. The familiarity ratings were used as the covariate. This was done to control for recall differences due to prior familiarity with the sentence content. Analyses were performed on overall recall and on recall with errors excluded. All significance tests on the means were done using the Newman-Keuls test, with $p<.05$.

Proposition recall. An ANCOVA on the mean proportion of the total definition-related and fact-related propositions recalled at each level by each subject indicated significant main effects of type $[F(1,33)=35.44, M S e=$ $.01, p<.001]$ and level $[F(2,67)=68.82, M S \mathrm{e}=.01$, $p<.001]$, and a significant type $\times$ group interaction $[F(1,33)=6.65, M S e=.01, p<.014]$. The type $\times$ group interaction is shown in Figure 2a. This interaction shows that the difference in the proportion of definition and fact propositions recalled is greater for novices than for experts. The adjusted cell means indicated that novices recalled .44 of the definition propositions and .30 of the fact propositions; experts recalled .48 of the definition propositions and .43 of the fact propositions. The difference in recall of definition and fact propositions is significant for the novices but not for the experts. Thus novices spent more time on definitions than on facts when reading the passages, and recalled significantly more definition- than fact-related information afterward. The difference between novice and expert recall was significant for facts but not for definitions.

The main effect of level is due to the fact that more target sentence propositions were recalled from the upper levels than from the lower levels of the passage hierarchies. All adjusted means were significantly different.

Propositions recalled accurately. Of an average total recall of 129 propositions from the target sentences, a mean of only 1.5 of these were recalled incorrectly. Because the number of recall errors was so low, the errors were not analyzed. Instead, the propositions recalled incorrectly were subtracted from the total recall data set, and the mean proportion of propositions recalled correctly by each subject were analyzed. The elimination of errors did not greatly alter the data set. The results of the anal- (a)

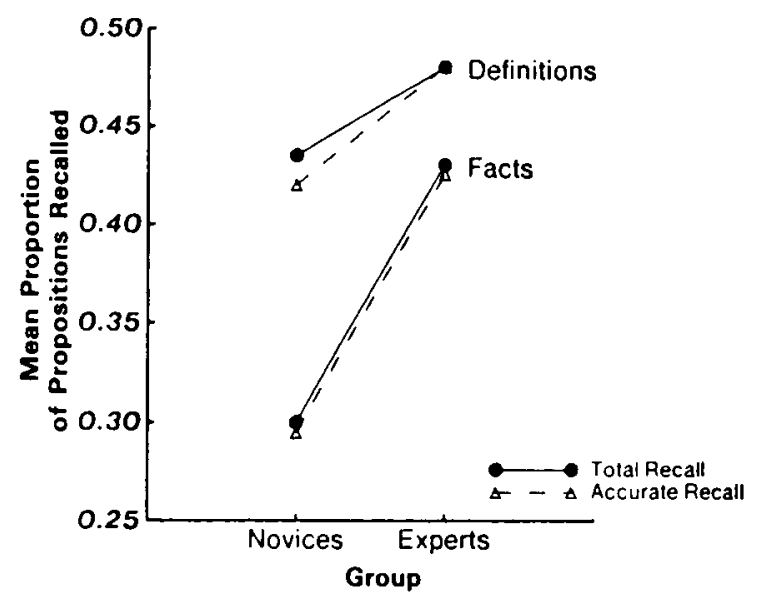

(b)

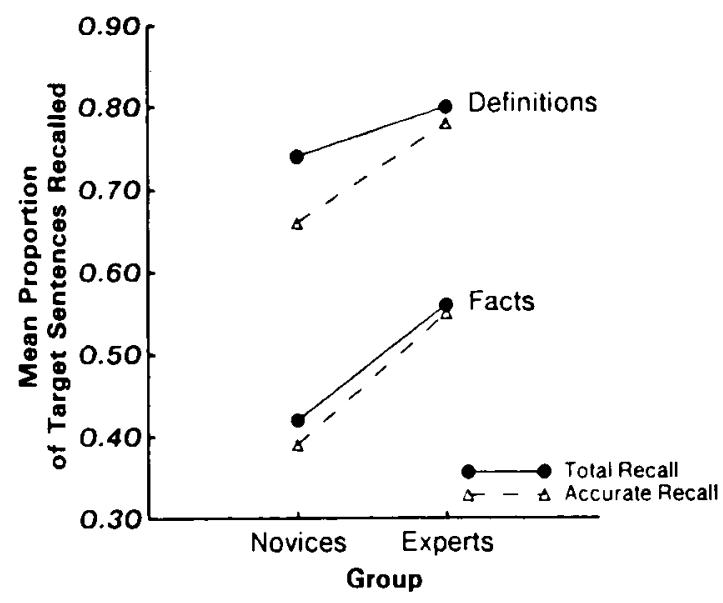

Figure 2. Mean proportion of (a) target-sentence propositions and (b) target sentences recalled by each group.

ysis indicated significant main effects due to type $[F(1,33)$ $=32.38, M S e=.01, p<.001]$ and level $[F(2,67)=$ $65.51, M S e=.01, p<.001]$, and significant interactions of type $\times$ group $[F(1,33)=5.74, M S e=.01, p<.022]$ and type $\times$ level $[F(2,67)=3.06, M S e=.01$, $p<.053]$. The pattern of means for the type $\times$ group interaction was similar to that of the total data set (see Figure 2a). The type $\times$ level interaction is due to the fact that recall declined with level more sharply for facts than for definitions. The adjusted mean proportions of definition propositions recalled accurately were .52 (level 1 ), .47 (level 2), and .37 (level 3 ). For facts, these mean were $.47, .40$, and .22 , respectively. All means were significantly different with the exception of definition recall at levels 1 and 2 .

Sentence recall. An ANCOVA on the mean proportion of definitions and facts recalled from each level by each subject indicated significant main effects of type $[F(1,33)=155.35, M S e=.03, p<.001]$ and level $[F(2,67)=95.96, M S e=.02, p<.001]$, and a significant type $\times$ group interaction $[F(1,33)=4.78$, 
MSe $=.03, p<.036]$. These are the same effects found in overall proposition recall.

The type $\times$ group interaction shows that the difference in the proportion of definitions and facts recalled was greater for the novices than for the experts. The adjusted means for this interaction are plotted in Figure $2 b$. Novices recalled a mean of .74 definitions and .42 facts; experts recalled a mean of .80 definitions and .56 facts. Both groups recalled a significantly greater proportion of definitions than facts, but the novices recalled significantly fewer facts than the experts. There was not a significant difference between experts and novices in recall of definitions. This interaction again suggests that the subjects were most likely to recall the type of information that they spent the most time on during reading.

More high-level than low-level target sentences were recalled by both groups. All adjusted means for the three levels were significantly different.

Sentences recalled accurately. All sentences recalled incorrectly were eliminated from the total sentence recall data, and an ANCOVA was performed on the proportion of target sentences recalled correctly. Most of the recall errors occurred in core propositions. Eliminating errors at the sentence level therefore produced a greater alteration of the data set than did dropping errors from the proposition data, because the errors composed a larger proportion of the sentence recall data. However, the absolute number of errors was still very small. Subjects recalled an average of 22.7 of the 36 target sentences, and incorrectly recalled an average of 1.2 of these.

The analysis of the mean proportion of target sentences recalled correctly by each subject from each level indicated significant main effects of type $[F(1,33)=104.43$, $M S e=.03, p<.001]$ and level $[F(2,67)=55.94$, $M S e=.03, p<.001]$, and a significant type $\times$ level interaction $[F(2,67)=5.74, M S e=.02, p<.005]$. There was not a significant type $\times$ group interaction in this data set $[F(1,33)=.71, M S e=.03, p<.41]$.

The mean proportion of definitions and facts recalled accurately by novices and experts is plotted in Figure $2 b$ for comparison with the type $\times$ group interaction found in the total sentence recall data. The greatest difference between the two data sets is in novice recall of definitions. The elimination of recall errors produced a more dramatic drop in the recall level for this cell than for any other. The fact that this did not occur with the proposition analyses indicates that novices did not recall more definition-related information incorrectly (relative to facts), but were more likely to recall incorrectly a core proposition from a definition. Thus the extra time spent on definitions resulted in more definitions being recalled, but not in more accurate recall. For a detailed analysis of the nature of the recall errors, see Dee-Lucas and Larkin (1987).

The type $\times$ level interaction is due to the fact that the greatest decline in definition recall occurs between levels 1 $(\mathrm{M}=.86)$ and $2(M=.67$; level $3, M=.63)$, whereas the largest decrease in fact recall occurs between levels $2(M=.50)$ and $3(M=.28 ;$ level $1, M=.63)$. There was no significant difference in the recall of definitions from levels 1 and 2; all other means were significantly different. As with the type $\times$ level interaction in proposition recall, the difference between the definition and fact recall is greatest at level 3 .

\section{DISCUSSION}

Although novices, by definition, do not have fully developed knowledge structures for unfamiliar domains, recent research has shown that novices do form a rudimentary type of content schema for scientific texts. This schema is in the form of rules that specify what types of information are important in these texts. Only beginninglevel students appear to possess these information-type rules; they are not found with naive subjects (i.e., those without domain-related training) or experts (Dee-Lucas \& Larkin, 1988). Thus these rules are the result of limited domain-related training. The current study investigated the extent to which this type of domain-specific knowledge is used by novices in processing science texts. It also compared the general study strategies of novices and experts in adjusting reading time according to passage familiarity and structure.

\section{Novice Rules and Text Processing}

The results reported here indicate that novice importance rules do influence attention during reading. Novices consider definitions to be more important than facts when judging importance (Dee-Lucas \& Larkin, 1986, 1988), and they spend more time on definitions than facts when studying physics texts. The use of this rule by novices was examined for both the initial reading and rereadings of the passages. The difference in reading times for definitions and facts was greatest on the first reading trial, indicating that these rules are "active"' on the initial reading of the passage. Apparently, novices devote extra time to definitions as soon as they are identified during reading. This is consistent with research that indicates that readers automatically update their text representation with important text content as they read (Cirilo \& Foss, 1980; Goetz, Reynolds, Schallert, \& Radin, 1983; Just \& Carpenter, 1980; Lorch, Lorch, \& Matthews, 1985).

Novice importance rules also appear to influence learning: novices recall more definitions than facts. However, most of the novice recall errors occurred in recall of definitions. This suggests that novices may rate definitions higher in importance and spend more time on them because they find them difficult to learn, and not necessarily because they believe definitions are particularly important. However, the difference in recall accuracy between definitions and facts is very small. Moreover, experts also rate definitions as more important for novices to learn (Dee-Lucas \& Larkin, 1986) and spend more time on definitions when they are reading physics texts, indicating that, on the average, definitions are more important for understanding physics. Additionally, novices rate definitions higher in importance even when the content of definitions and facts is identical, and therefore equally difficult 
(Dee-Lucas \& Larkin, 1988). Thus novice importance rules appear to partially reflect the nature of the content of physics, rather than to relate solely to learning difficulty.

Although there was a significant difference between recall of definitions and facts, recall differences may be somewhat attenuated because subjects read the passages three times. The subjects were able to concentrate on the facts and other types of information that they considered to be less critical on the second and third readings of the passages, possibly bringing the recall level for facts closer to that of definitions. The type $\times$ trial interaction in the reading time data suggests that subjects may have adopted this strategy. There was a greater decline in reading time over trials for definitions than for facts, indicating that the amount of attention given to facts increased relative to that given to definitions with subsequent passage readings.

The current study indicates that differences between the content schema of novices and that of experts are reflected in differences in the actual processing of domain-related texts. Thus novice rules are part of the control schema (Kintsch, 1982) used by novices to govern their text processing. The use of these importance rules during comprehension can be viewed as a "macrostrategy" within the framework of the Kintsch and van Dijk (1978; van Dijk \& Kintsch, 1983) model of comprehension. According to this model, an integral part of the comprehension process is the formation of a "macrostructure" for the text (see also Guindon \& Kintsch, 1984). The macrostructure is a representation of the gist or main ideas of the passage and is derived from the information in the text through the use of macrorules, which select from and reduce the text content. Macrostrategies are the sets of strategies used by readers in applying macrorules to construct a macrostructure. The use of novice informationtype rules constitutes such a strategy in that these rules provide a basis on which to select the important text content.

Although this study indicates that novices use information-type importance rules as a macrostrategy in the naturalistic "study setting" provided, it is quite possible that they would use other rules under other circumstances. For example, under strong instructional sets, such as "learn all the formulas in this text," the influence of these rules would be attenuated or eliminated. However, these rules do appear to be part of the novice "default" strategy. That is, when novices are studying a physics text, they tend to expect certain types of information to be important for full understanding of that text. Moreover, these importance rules are very robust: they have been found in tasks requiring subjects to rate the importance of information, select the important content from texts, write summaries, and (in the current study) read and recall. They have also been replicated over many different novice groups (see Dee-Lucas \& Larkin, 1986, 1988).

The use of these rules is a very sensible default strategy. As noted earlier, experts also judge definitions as more important than facts, and spend more time on definitions than on facts. This suggests that definitions are typically important for understanding this content domain, and lack of attention to definitions may, in fact, result in comprehension difficulties. The problem with using this type of rule is that it is too general for accurately identifying what is important.

The experts and novices in this study were graduate students and undergraduates, respectively. These are the two subject groups that were used in previous research on novice importance rules. These groups were chosen in order to compare people who learn from physics texts (i.e., undergraduates) with people who use them in teaching (i.e., graduate students) to see if the groups match in terms of what they consider to be important to learn from physics texts (Dee-Lucas \& Larkin, 1986). Because the current study attempted to replicate previous research findings using different dependent measures, the same subject groups were chosen. It is possible that the difference between these two groups in the time spent on definitions and facts was due to differences in their sensitivity to rhetorical indicators of importance, as opposed to differences in the perceived importance of different categories of information. That is, if the passages unintentionally signaled definitions as being more important than facts through the use of rhetorical devices, and if novices were more influenced than experts by these rhetorical signals during reading, then they would spend (relative to experts) more time on definitions and less time on facts. However, the fact that hierarchical structure had the same effect on the importance judgments of novices and experts suggests that the two groups did not differ in sensitivity to rhetorical indicators of importance. Additionally, previous research controlling the content and location of definitions and facts while varying only category membership shows that novices judge definitions as more important than facts when rhetorical indicators of importance are held constant (Dee-Lucas \& Larkin, 1988). Furthermore, this earlier research compared the importance judgments of graduate students and undergraduates who were equivalent in physics background and found no difference between these two groups in the judged importance of definitions and facts, indicating that the definition-fact difference between experts and novices is due to differences in physics experience rather than differences in educational level.

\section{Expert and Novice Reading Strategies}

Although this study focused on the role of novice importance rules in text processing, it also permits a comparison of expert and novice strategies in rereading passages over several study trials. Both experts and novices appear to have adopted a "learning-to-criterion" strategy in varying their reading times according to sentence type and reading trial. There was very little difference between the groups in this respect. Subjects decreased the extra processing given to definitions over reading trials, so that the difference in the reading times for definitions and facts was very small by the third reading of the passage. Thus subjects appear to have concentrated on the most impor- 
tant passage content on the first reading, and then gradually decreased the extra processing given to that content as it was more thoroughly learned. Although reading times decreased for facts as well, the decrease was not as rapid, so subjects were in effect increasing the amount of attention given to facts relative to that given to definitions with the passage rereadings. Although definitions are considered more important than facts, facts are also judged above average in importance (Dee-Lucas \& Larkin, 1986, 1988), so it is reasonable to expect subjects to attend to both sentence types.

Although both experts and novices appear to have adopted similar strategies in learning the two sentence types, they differed in the extent to which they adjusted reading times according to reading trial and passage. Experts decreased their reading time more sharply over trials than did novices. This suggests that their greater expertise enabled them to learn the passage content more efficiently. This efficiency was also indicated by the fact that they recalled more target sentences overall than did novices, but read the target sentences more quickly. This is consistent with research that indicates that expertise facilitates learning from domain-related texts (Chiesi et al., 1979; Spilich et al., 1979). Spilich et al. found that baseball experts remembered more than novices after reading a passage describing a baseball game. They attributed the superior recall of the experts to their more elaborated and differentiated knowledge system, which allowed them to map more text content onto preexisting knowledge structures. The ease with which experts learned the content of physics texts in the current study is also most likely due to a large overlap between existing knowledge and text content. The experts could simply tag or elaborate existing knowledge structures in constructing a representation of the text, whereas novices had to generate new knowledge structures. Thus the experts could decrease reading time over trials more sharply than could novices because they did not need as thorough a review of the material in order to check that the appropriate knowledge had been tagged or to tag additional content. Novices, on the other hand, most likely used the rereadings to check, elaborate, and further generate new knowledge structures, tasks that are more time-consuming (see Johnson \& Kieras, 1983).

Experts also distinguished between the two passages to a greater degree than did novices in adjusting their reading times. Although familiarity and comprehensibility differences at the sentence level were controlled through subject ratings, there was an additional effect of passage on the reading times. Both experts and novices spent more time on target sentences in one passage, but the experts spent more time on this passage and less time on the other passage than the novices. Because the two passages differ on a number of variables, the source of this effect is not clear. However, because the main effect of passage on reading time correlates with overall topic familiarity (with both groups spending more time on the less familiar pas- sage), it suggests that general topic familiarity may have influenced reading times. If this is the case, then the passage $\times$ group interaction indicates that the experts were better able to adjust study time in accordance with passage topic, as well as reading trial. Thus the more elaborated knowledge structures of the experts may have allowed them to better determine how much study time was needed to master the content related to each topic.

This study controlled for passage structure in order to determine whether the influence of novice importance rules varied with hierarchical level. It was found that more time was spent on definitions than facts at all levels of the passage hierarchies. However, hierarchical level did have a main effect on readers' attention. Both experts and novices spent more time on high-level than low-level target sentences, and also recalled more high-level content. As discussed previously, novices are very adept at using various rhetorical indicators of importance, including passage structure (Kieras, 1985; Meyer, 1983). Thus, in the current study, novices were able to distinguish low-level elaborative facts and definitions from those that provided the main points of the passage. Therefore, novices apparently spent more time on definitions and less time on facts at all levels relative to the experts, with level modifying attention in a similar manner for both groups. This is consistent with the findings from the importance judgment research that indicate that novices judge high-level definitions and facts as more important than low-level content, but judge definitions overall higher in importance and facts overall lower in importance than do experts.

Although levels effects in reading time have been reported in other research (Cirilo \& Foss, 1980; Schmalhofer \& Glavanov, 1986), Schmalhofer and Glavanov found that this effect could be altered with instructional sets that implicitly indicated as important content lower in the passage structure. Thus levels effects in reading times appear to be a specific instance of the general principle that readers attend to the important text information, regardless of whether importance is defined on the basis of instructions, learning goals, text features, or schematic knowledge (see Anderson, 1982). This effect in reading times suggests that the influence of level on recall is due to selective attention: readers recognize highlevel content as important, and therefore devote extra effort to learning it, resulting in longer reading times and better recall. However, this does not preclude alternative accounts of the levels effect in recall that do not rely on a selective attention hypothesis. The Kintsch and van Dijk (1978) model of comprehension attributes the effect to the repeated reprocessing received by high-level content as readers integrate low-level content with the high-level information. Additionally, Britton (Britton, Meyer, Hodge, \& Glynn, 1980; Britton, Meyer, Simpson, Holdredge, \& Curry, 1979) proposed several accounts of the levels effect which rely on retrieval mechanisms. It may well be the case that all of these phenomena contribute to levels effects in recall. 


\section{Conclusion}

This research compared the effects of several variables on the attention and recall of novice and expert physicists, focusing specifically on sensitivity to information-type categories. The findings indicated that sentence category does influence the text processing of novices, with novices differentiating between category types to a greater degree than experts in adjusting their reading times. This is the same pattern found in importance judgments and summaries, and is also reflected in what novices remember from texts.

Given that novice importance rules influence attention and learning from text, the accuracy of these rules has important implications for the efficiency with which novices master text content. The fact that there is a partial mismatch between the content being learned by novices and that considered important for mastery by experts indicates that novices are attending to unimportant information (possibly learning this at the expense of other content) and missing some important content.

The findings from this study suggest that text writers for novice audiences will be most effective in enhancing learning if they use knowledge of subject-based content schema in conjunction with rhetorical devices in signaling the important text content. Writers need to know not only what is important in the text subject matter, but also what their readers think is important in order to be most effective in aiding learning of the critical text content.

\section{REFERENCES}

ANDERSON, R. C. (1982). Allocation of attention during reading. In A. Flammer \& W. Kintsch (Eds.), Discourse processing (pp. 292305). Amsterdam: North-Holland.

Britton, B. K., Meyer, B. J. F., Hodge, M. H. , GlynN, S. (1980). Effects of the organization of text on memory: Tests of retrieval and response criterion hypotheses. Joumal of Experimental Psychology: Human Learning \& Memory, 6, 620-629.

Britton, B. K., Meyer, B. J. F., Simpson, R., Holdredge, T. S., - CURRY, C. (1979). Effects of the organization of text on memory: Tests of two implications of a selective attention hypothesis. Joumal of Experimental Psychology: Human Leaming \& Memory, 5, 496-506.

Chiesi, H., SPILICH, G., Voss, J. (1979). Acquisition of domainrelated information in relation to high and low domain knowledge. Journal of Verbal Learning \& Verbal Behavior, 18, 257-274.

CIRILo, R., Foss, D. (1980). Text structure and reading time for sentences. Joumal of Verbal Leaming \& Verbal Behavior, 19, 96-109.

DeE-Lucas, D., Larkin, J. H. (1986). Novice strategies for comprehending scientific texts. Discourse Processes, 9, 329-354.

DEE-LuCAS, D., LARKIN, J. H. (1987). Attentional strategies for studying scientific texts (Tech. Rep. No. A.C.P. 17). Pittsburgh, PA: Camegie-Mellon University, Department of Psychology.

Dee-Lucas, D., \& Larkin, J. H. (1988). Novice rules for assessing importance in scientific texts. Joumal of Memory \& Language, 27, 288-308.

Goetz, E. T., Reynolds, R. E., Schallert, D. L., Radin, D. I. (1983). Reading in perspective: What real cops and pretend burglers look for in a story. Joumal of Educational Psychology, 75, 500-510.

Graesser, A. C., Hofrman, N. L., Clark, L. F. (1980). Structural components of reading time. Journal of Verbal Learning \& Verbal Behavior, 19, 131-151.

Guindon, R., Kintsch, W. (1984). Priming macropropositions: Evidence for the primacy of macropropositions in the memory for text. Journal of Verbal Learning \& Verbal Behavior, 23, 508-518.
JoHNSON, W., KIERAS, D. E. (1983). Representation-saving effects of prior knowledge in memory for simple technical prose. Memory \& Cognition, 11, 456-466.

Just, M. A., CARpenter, P. A. (1980). A theory of reading: From eye fixations to comprehension. Psychological Review, 87, 329-354.

KiERAS, D. E. (1980). Initial mention as a signal to thematic content in technical passages. Memory \& Cognition, 8, 345-353.

KiERAS, D. E. (1985). Thematic processes in the comprehension of technical prose. In B. K. Britton \& J. B. Black (Eds.), Understanding expository text (pp. 89-107). Hillsdale, NJ: Erlbaum.

KiERAS, D. E., BovaIR, S. (1981). Strategies for abstracting main ideas from simple technical prose (Tech. Rep. No. 9). Tucson: University of Arizona, Department of Psychology

KINTSCH, W. (1974). Representation of meaning in memory. Hillsdale, NJ: Erlbaum.

KinTsCH, W. (1982). Memory for text. In A. Flammer \& W. Kintsch (Eds.), Discourse processing (pp. 186-204). Amsterdam: North Holland.

Kintsch, W. VAN DiJk, T. A. (1978). Toward a model of text comprehension and production. Psychological Review, 85, 363-394.

LoRch, R. F., LoRch, E. P., Matthews, P. D. (1985). On-line processing of the topic structure of a text. Joumal of Memory \& Language, 24, 350-362.

MEYER, B. J. F. (1975). The organization of prose and the effect on recall. Amsterdam: North-Holland.

MEYER, B. J. F. (1983). Text dimensions and cognitive processing. In H. Mandl, N. Stein, \& T. Trabasso (Eds.), Leaming and understanding from text (pp. 3-47). Hillsdale, NJ: Erlbaum.

SChmalhofer, F., Glavanov, D. (1986). Three components of understanding a programmer's manual: Verbatim, propositional, and situational representation. Joumal of Memory \& Language, 25, 279-294.

Spilich, G. J., Vesonder, G. T., Chiesi, H. L., \& Voss, J. F. (1979). Text processing of domain-related information for individuals with high and low domain knowledge. Journal of Verbal Learning \& Verbal Behavior, 18, 275-290.

VAN Dijk, T. A., \& KinTsCH, W. (1983). Strategies of discourse comprehension. New York: Academic Press.

\section{NOTES}

1. The goal of Dee-Lucas and Larkin's (1986) research was to determine whether novices (i.e., physics students) agreed with experts (i.e., physics instructors) on what types of information are important for novices to learn in these texts. The experts were instructed to indicate the information in physics passages that they considered most important for their students to leam. Novices were told to indicate what they thought was most important to learn if they were going to be tested on the passage content.

2. The familiarity ratings were used to control for within-subject variations in prior knowledge of the definitions and facts. Johnson and Kieras (1983) showed that within-subject prior-knowledge differences measured by familiarity ratings are related to reading time. However, Johnson and Kieras collected familiarity ratings on the first reading of the passage and recorded reading times on the second passage reading. $\mathrm{Be}$ cause of the possibility of contaminating the reading time data for definitions and facts by having subjects read them first to rate their familiarity, the current study reversed this procedure and had subjects perform post hoc ratings of their preexperimental knowledge. This risked weakening the validity of the measure, in that subjects might judge the content as more familiar than it actually had been at the time of the initial reading. However, this would be indicated in the data analyses by a weak (or nonexistent) effect of familiarity on sentence reading times.

3. These reading times were calculated under the assumption that the sentences were very comprehensible and very familiar (i.e., familiar ity and comprehensibility ratings of 1). This level of adjustment was selected for these two variables because the mean familiarity and com prehensibility ratings given to the target sentences indicate that subjects generally found the sentences to be easy to understand and familiar in content. 


\section{APPENDIX}

The following is one of the passages used in this study. The target sentences are presented in bold, and the sentence type and hierarchical level are noted.

\section{FLUID STATICS}

In the preceding chapters, we studied the basic concepts needed to deal with systems consisting of many particles.

Hence, we can now discuss some of the important properties of common materials (such as solids, liquids and gases), which consist of many atoms or molecules.

We will spend most of our time discussing fluids (i.e., liquids and gases) because these have some remarkable properties of great importance for the understanding of physical and biological processes.

\section{Pressure}

Imagine a fluid in a container and in contact with some surface.

Assume that the fluid and any other relevant objects (such as the container) are at rest so that the fluid is in equilibrium.

When this is the case, the fluid in contact with any surface exerts a contact force perpendicular to the surface and the surface exerts an equal and opposite force on the fluid. (fact, level 1)

These two forces must be perpendicular to the surface or the fluid layers will not be motionless.

The force exerted by a fluid on any object can be described by specifying the "pressure," which is defined as the magnitude of a fluid force divided by the area of the surface on which it acts. (definition, level 1) This is a very useful concept for discussing fluid forces.

The amount of force produced by a fluid varies with surface area. (fact, level 2)

This force is exerted by molecules which are distributed over the entire surface exerting the pressure. (fact, level 3)

Therefore, if an area under pressure were made twice as big, for example, the number of molecules exerting a force would double and the total force exerted would become twice as large.

However, according to our definition, pressure is not proportional to area. If the area of the surface is made twice as big, the amount of pressure, which is equal to force divided by area, remains the same.

Although all fluids exert pressure, the pressure of the air in the atmosphere near the earth's surface is of special interest because we usually work in an environment of air at atmospheric pressure.

We can define atmospheric pressure at any point as the ratio of the weight of a column of air extending from that point to the top of the atmosphere, divided by the cross-sectional area of that column. (definition, level 2)

According to our definition, atmospheric pressure decreases with increases in altitude as the weight of the air above that point decreases. Atmospheric pressure also varies from day to day due to changes in air temperature: (fact, level 3)

Pressure is often expressed in terms of "gauge pressure," which is defined as the difference between the pressure at a point in a fluid and the atmospheric pressure. (definition, level 2)

The pressure at a point in a fluid is called the "absolute pressure," and is defined as simply the actual pressure at that point. (definition, level 3)

Since we live and work in an environment at atmospheric pressure, gauge pressure uses atmospheric pressure as a standard against which the actual pressure is compared.

For example, a mercury-filled manometer is commonly used to measure the difference between arterial blood pressure and the atmospheric pressure.
The measured blood pressure is then the gauge pressure of the blood. According to our definition of gauge pressure, the gauge pressure is negative when the absolute pressure is less than the standard atmospheric pressure.

\section{Density}

Any small portion of a material has some volume and some mass (which is the sum of the masses of all the atoms contained in this portion). If this portion is small enough, the number of atoms in it, and thus also its mass, is proportional to its volume. (fact, level 1)

For example, if the volume of a portion of water was increased so that it was 3 times as large, the number of atoms in this portion, and thus also the mass of this portion, would be 3 times as large.

We can specify a ratio called "density," which is defined as the mass of a portion of material divided by its volume, or mass per unit volume. (definition, level 1)

The density of a substance does not change with changes in volume. (fact, level 2)

Properties with this characteristic are called "intrinsic" quantities, which are defined as quantities which are independent of volume. (definition, level 3)

The density of a substance often varies with the position of the portion of the material taken, since there are often variations in the internal characteristics of substances. (fact, level 2)

However, density does not always depend on position of material sampled.

Some materials are "homogeneous," which is defined to mean that all intrinsic properties of the material are the same for every portion of the material. (definition, level 3)

Thus, in materials which are homogeneous, the density is the same at each point.

It is sometimes convenient to re-express density in terms of specific gravity.

Specific gravity is defined as the ratio of the density of a substance to the density of water. (definition, level 2)

For example, pure water has a specific gravity of exactly 1 , while mercury has a specific gravity of 13.6.

The specific gravity of a substance varies with its temperature. (fact, level 3)

Therefore, a specific gravity value for a substance is accompanied by a statement of the temperature at which it was evaluated.

\section{Buoyant Force}

Suppose an object is surrounded by a liquid at rest, and this object, having a density less than the density of the liquid, floats partly submerged in this liquid.

The pressure exerted by the fluid on each part of the surface of this submerged body does not depend on the material that the body is made of. (fact, level 1)

If this hypothetical object is replaced by fluid similar to that of its surroundings, for example, this body of fluid will experience the same pressures that acted on the immersed object, and will be at rest.

An object floating or submerged in a liquid experiences a "buoyant force" due to the surrounding liquid, which is defined as the product of the density of the liquid, the gravitational acceleration $\mathrm{g}$, and the volume $V$, of the liquid displaced by the object. (definition, level 1) Thus the buoyant force is simply the total force exerted on the object by the surrounding fluid.

(Manuscript received February 4, 1987; revision accepted for publication December 1, 1987.) 